\title{
Correction to: A multi-mode Wnt- and stemness-regulatory module dictated by FOXM1 and ASPM isoform I in gastric cancer
}

\author{
Chung-Chi Hsu ${ }^{1}$. Wen-Ying Liao ${ }^{2}$. Kwang-Yu Chang ${ }^{3} \cdot$ Tze-Sian Chan $^{2,4,5,6} \cdot$ Po-Jui Huang ${ }^{2,5,6}$. Chun-Ting Chiang ${ }^{7}$. \\ Yan-Shen Shan ${ }^{8} \cdot$ Lin-Hsin Cheng $^{2} \cdot$ Tai-Yan Liao $^{2} \cdot$ Kelvin K. Tsai ${ }^{2,3,5,6,9,10}$
}

Published online: 26 March 2021

(c) The International Gastric Cancer Association and The Japanese Gastric Cancer Association 2021

\section{Correction to: Gastric Cancer}

https://doi.org/10.1007/s10120-020-01154-5

In the original publication of the article a blot in Fig. $6 \mathrm{~b}$ (IB: b-catenin) was missed during the production process. The correct Fig. 6 is given in this correction. The publisher regrets for the error occurred.

The original article has been corrected.

Publisher's Note Springer Nature remains neutral with regard to jurisdictional claims in published maps and institutional affiliations.

The original article can be found online at https://doi.org/10.1007/ s10120-020-01154-5.

Kelvin K. Tsai

tsaik@tmu.edu.tw

1 School of Medicine, College of Medicine, I-Shou University, Kaohsiung City 824410, Taiwan

2 Laboratory of Advanced Molecular Therapeutics, Graduate Institute of Clinical Medicine, College of Medicine, Taipei Medical University, 250 Wuxing St., Xinyi Dist., Taipei City 110301, Taiwan

3 National Institute of Cancer Research, National Health Research Institutes (NHRIs), Tainan City 704016, Taiwan

4 School of Medicine, College of Medicine, Taipei Medical University, Taipei City 110301, Taiwan

5 Division of Gastroenterology, Wan Fang Hospital, Taipei Medical University, Taipei City 110301, Taiwan
6 Integrative Therapy Center for Gastroenterological Cancers, Wan Fang Hospital, Taipei Medical University, Taipei City 110301, Taiwan

7 Department of Pathology, National Cheng-Kung University Hospital, Tainan City 704302, Taiwan

8 Department of Surgery, National Cheng-Kung University Hospital, Tainan City 704302, Taiwan

9 TMU Research Center of Cancer Translational Medicine, Taipei Medical University, Taipei City 110301, Taiwan

10 TMU and Affiliated Hospitals Pancreatic Cancer Group, Taipei Medical University, Taipei City 110301, Taiwan 
Fig. 6 FOXM1-mediated $\beta$-catenin/Wnt activation and GC stemness requires ASPMiI. a IB analysis showing that $\mathrm{OE}$ of $D V L 3$ significantly restored the protein abundance level of $\beta$-catenin in FOXM1-deficient NCI-N87 cells. b OE of FOXMI failed to rescue the protein abundance levels of $\beta$-catenin and DVL3 in ASPMiI-deficient NCI-N87 cells. c Wnt-specific luciferase activity in NCI-N87 cells with OE of FOXM1 with or without the concurrent $\mathrm{KD}$ of $A S P M \mathrm{v} 1$ expression (mean $\pm \mathrm{SEM}, N=3$ biological replicates with two technical replicates). $* * * P<0.001, t$ test. NS, not significant. d LDA demonstrating the tumorsphereforming efficacy of NCI-N87 cells with control KD, ASPMv1 KD or FOXM1 KD with or without the concurrent $\mathrm{OE}$ of FOXM1 (maximum likelihood estimates with $95 \%$ confidence interval, $N=4$ biological replicates with two technical replicates) a

$$
\text { IB: DVL3 }
$$

IB: $\beta$-catenin

IB: $\beta$-tubulin

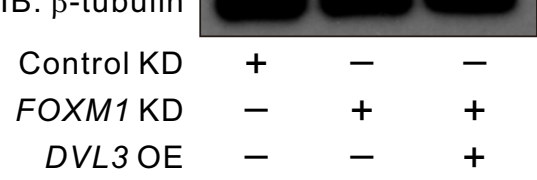

C

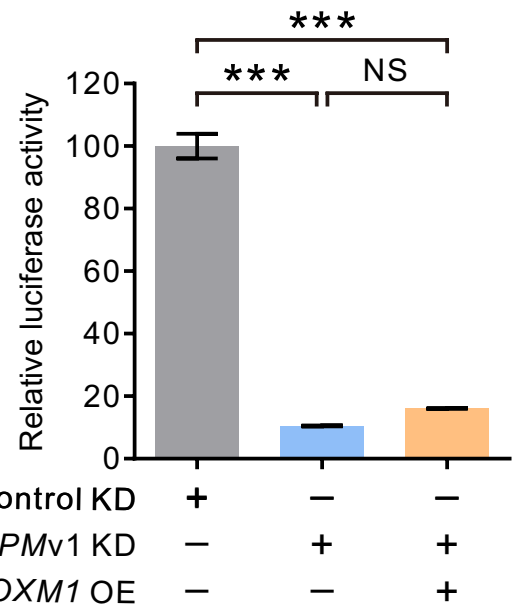

b

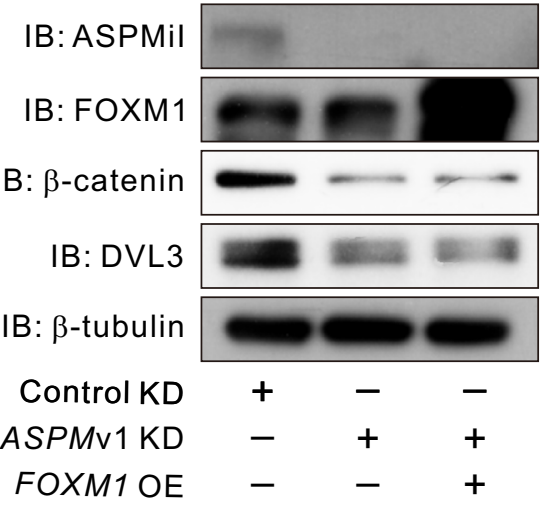

d

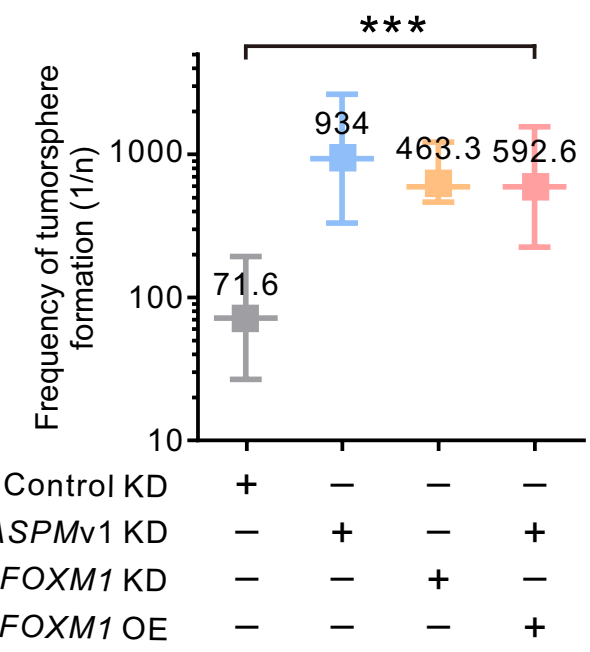

